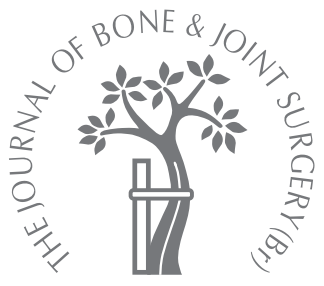
A. J. Price,
P. T. Oppold,
D. W. Murray,
A. B. Zavatsky
From the Nuffield
Orthopaedic Centre,
Oxford, England

\section{Simultaneous in vitro measurement of patellofemoral kinematics and forces following Oxford medial unicompartmental knee replacement}

The Oxford medial unicompartmental knee replacement was designed to reproduce normal mobility and forces in the knee, but its detailed effect on the patellofemoral joint has not been studied previously. We have examined the effect on patellofemoral mechanics of the knee by simultaneously measuring patellofemoral kinematics and forces in $\mathbf{1 1}$ cadaver knee specimens in a supine leg-extension rig. Comparison was made between the intact normal knee and sequential unicompartmental and total knee replacement. Following medial mobile-bearing unicompartmental replacement in 11 knees, patellofemoral kinematics and forces did not change significantly from those in the intact knee across any measured parameter. In contrast, following posterior cruciate ligament retaining total knee replacement in eight knees, there were significant changes in patellofemoral movement and forces.

The Oxford device appears to produce near-normal patellofemoral mechanics, which may partly explain the low incidence of complications with the extensor mechanism associated with clinical use.

The design of a knee prosthesis will affect its kinematic and kinetic performance. ${ }^{1,2}$ Features of the design include the shape of the components, the constraint of the articulation and the retention of ligaments or soft tissues around the knee. Total knee replacement (TKR) produces good clinical results, but because of the loss of ligaments and changes to surface geometry, it does not restore normal tibiofemoral or patellofemoral mechanics to the knee. ${ }^{3-9}$ Abnormal patellofemoral movement and forces have been implicated as a factor in the incidence of complications of the extensor mechanism following TKR. ${ }^{10}$

The Oxford medial unicompartmental knee replacement (UKR) was designed to allow near-normal movements of the knee. ${ }^{11}$ The device retains all ligaments, employs a spherical femoral component, a flat tibial base-plate and a fully congruous meniscal bearing. There is evidence from mathematical modelling as well as studies in the cadaver and in vivo that it can reproduce some aspects of normal kinematics. ${ }^{2,12,13}$ However, no study has examined in detail how the device affects the kinematics and kinetics of the patellofemoral joint, although clinical observation suggests that few complications of the extensor mechanism are seen with its use. ${ }^{14-19}$
The aim of this study was to measure the effect of implantation of an Oxford medial UKR on patellofemoral mechanics by simultaneously measuring patellofemoral kinematics and forces in an in vitro cadaver model.

\section{Materials and Methods}

A total of 11 post-mortem specimens of the human knee were tested in a supine legextension rig which held the femur rigidly at $45^{\circ}$ to the horizontal and allowed the tibia to hang vertically under its own weight. A $3 \mathrm{~kg}$ mass was hung from the tibia $0.3 \mathrm{~m}$ from the centre of the knee joint to create a flexion moment. A force was applied through the rectus femoris tendon to balance the flexion moment and to flex and extend the knee specimen slowly through $0^{\circ}$ to $120^{\circ}$. The leg-extension rig was used to test the 11 intact knees, with subsequent measurements taken from the same knees after sequential implantation of a medial mobile-bearing UKR (Oxford Phase 2; Biomet UK Ltd, Swindon United Kingdom, $\mathrm{n}=11)$ and a posterior cruciate ligament (PCL)-retaining TKR (AGC, tibiofemoral components only; Biomet UK Ltd, $\mathrm{n}=8$ ).

Retroflective spherical markers were attached to the tibia, femur and patella and to the proximal and distal ends of the patellar tendon. The movements of the markers were
$J$ Bone Joint Surg $[B$

Received 21 June 2006

Accepted 8 August 2006 


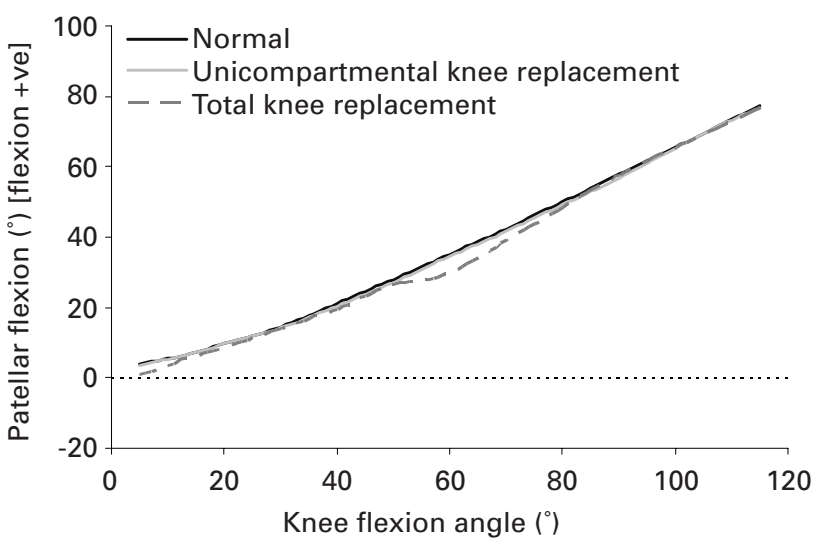

Fig. 1

Mean patellar flexion plotted against knee flexion angle.

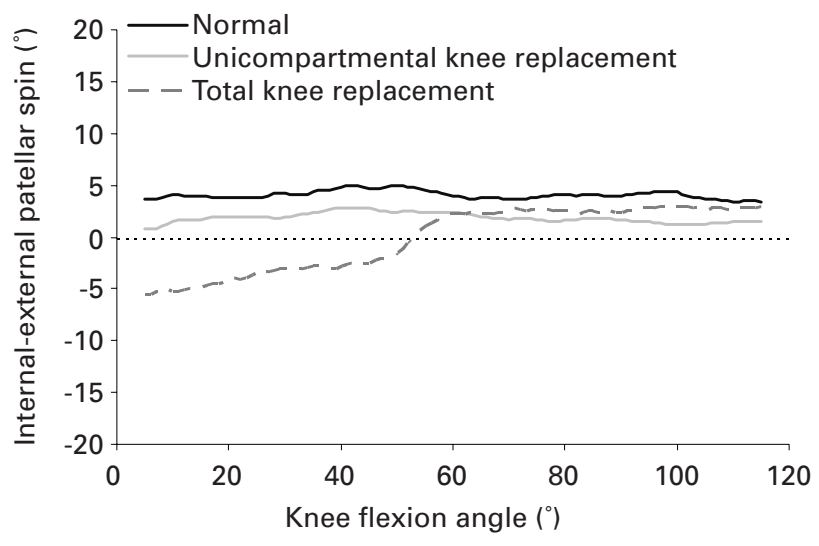

Fig. 3

Mean internal-external patellar spin plotted against knee flexion angle.

tracked by a five-camera Vicon 370 system (Vicon Motion Systems, Oxford, United Kingdom). A uniaxial force transducer (Transducer World, Aylesbury, United Kingdom) was used to measure the simulated quadriceps force. A sixdegree-of-freedom force transducer, custom-built at the Oxford Orthopaedic Engineering Centre, based closely on the design of Singerman et al, ${ }^{20}$ was used to measure the three forces and three moments acting upon the posterior surface of the patella.

Anatomically-based coordinate systems were defined in the femur, tibia and patella. Joint rotations were calculated in terms of joint coordinate system angles. ${ }^{21}$ For the tibiofemoral joint, the rotations were: flexion-extension about a mediolateral axis in the femur, internal-external tibial rotation about the long axis of the tibia, and abductionadduction about a third axis running approximately anteroposteriorly. For the patellofemoral joint, the rotations were: flexion about a mediolateral axis in the femur, mediolateral tilt about a proximodistal axis in the patella, and internalexternal rotation (or spin) about a third axis running

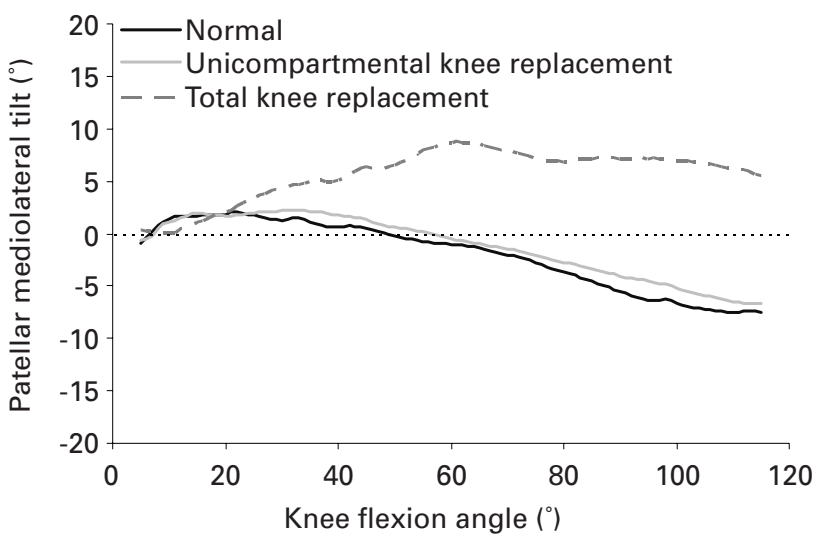

Fig. 2

Mean patellar mediolateral tilt plotted against knee flexion angle.

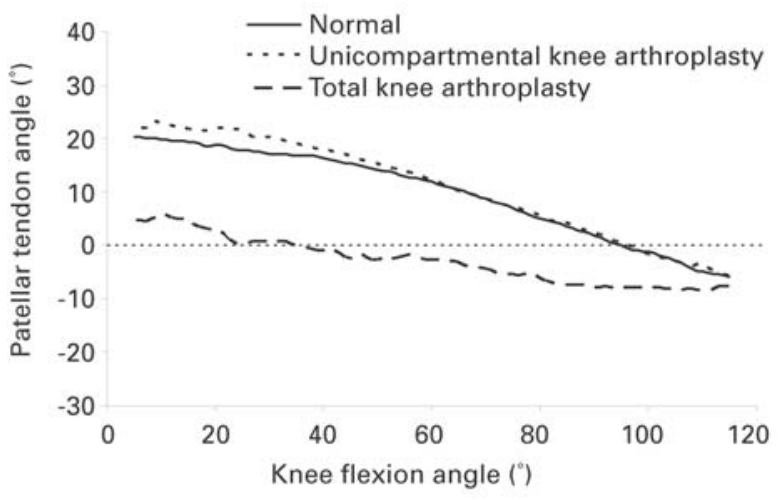

Fig. 4

Mean patellar tendon angle plotted against knee flexion angle.

approximately anteroposteriorly. The angle between the patellar tendon and the long axis of the tibia was also calculated.

Full details of the transducer calibrations and data analysis, along with results for the intact state of the knee, have been published previously, showing the method to be accurate, reliable and internally valid. ${ }^{22}$

The data are presented here are for extension of the knee specimens from $120^{\circ}$ to $0^{\circ}$ of tibiofemoral flexion. A twoway repeated-measures analysis of variance with a significance level of 0.05 was used to compare the different states of the knee when intact, with a UKR and after TKR. When more than one state was compared, a post hoc Tukey ${ }^{23}$ test was employed to distinguish the location of any statistically-significant differences.

\section{Results}

The results are presented as means with standard deviations. As the intact knee flexed up to $115^{\circ}$, the tibia rotated internally by a mean of $5^{\circ}$ (SD $12^{\circ}$ ) and abducted by $5^{\circ}$ (SD 


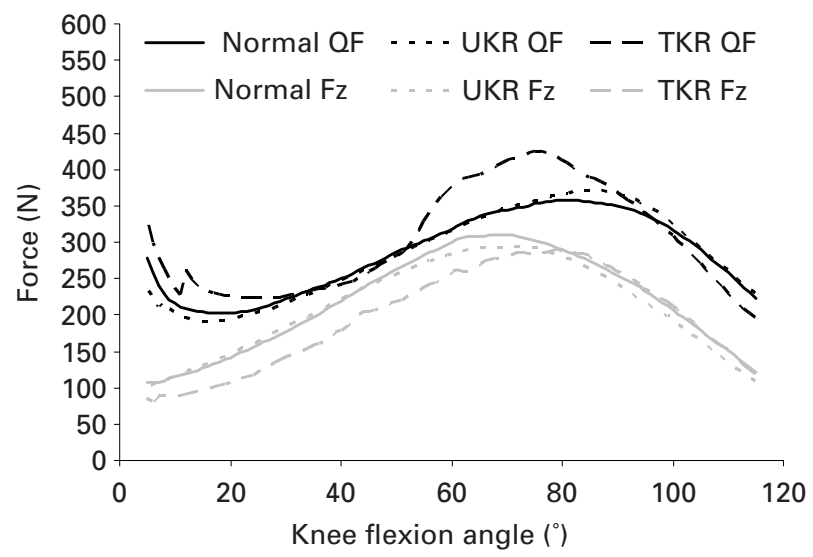

Fig. 5

Mean quadriceps force (QF) and anterior-posterior compressive force (Fz) plotted against knee flexion angle.

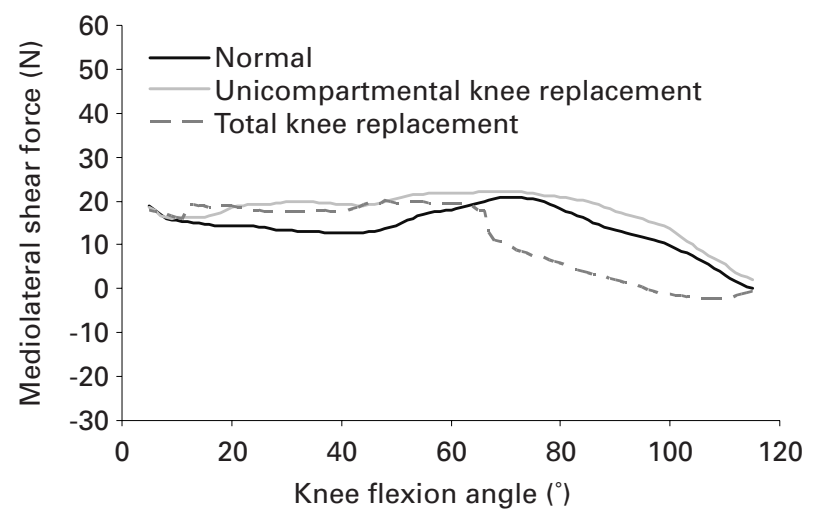

Fig. 6

Mean mediolateral shear force plotted against knee flexion angle.

$\left.9^{\circ}\right)$. At the same time, patellar flexion increased evenly to a mean of $78^{\circ}$ (SD $6^{\circ}$ ) (Fig. 1), the patella rotated internally by $3^{\circ}\left(\mathrm{SD} 10^{\circ}\right)$ and tilted laterally by $6^{\circ}$ (SD $10^{\circ}$ ) (Figs 2 and 3 ). The angle between the patellar tendon and the long axis of the tibia decreased as tibiofemoral flexion increased (Fig. $4)$, and the quadriceps force reached its mean maximum value of $356 \mathrm{~N}$ (SD 42.8) at $80^{\circ}$ of tibiofemoral flexion (Fig. 5 ). The mean anteroposterior component of the patellofemoral force reached a maximum of $309 \mathrm{~N}$ (SD 37.0) compression at $73^{\circ}$ of tibiofemoral flexion (Fig. 5).

After implantation of the mobile-bearing UKR there were no significant changes in the measured kinematics and forces. Tibiofemoral adduction/abduction and axial rotation followed a similar pattern of movement to the intact knee, although there was a reduction in total tibial axial rotation. The patellofemoral kinematics (Figs 1 to 3 ), the patellar tendon angle (Fig. 4), the patellofemoral forces

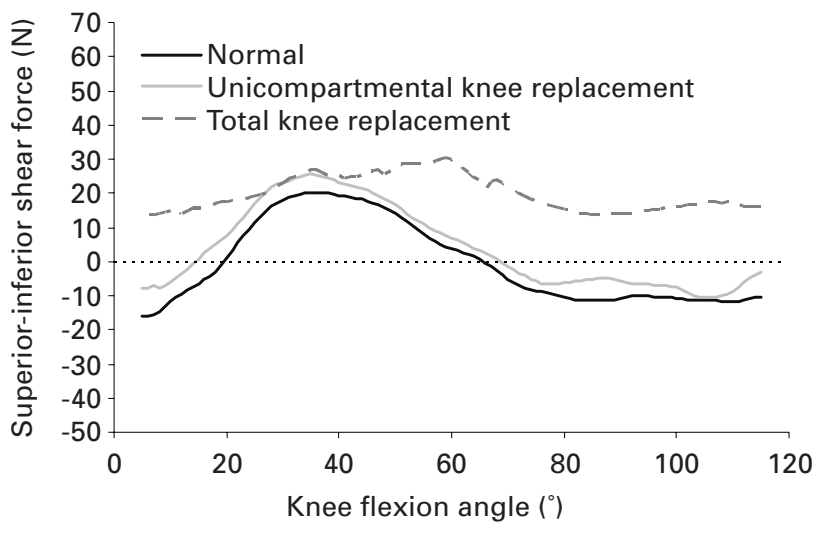

Fig. 7

Mean superior inferior shear force plotted against knee flexion angle.

(Figs 5 to 7), and the quadriceps force (Fig. 5) did not change significantly from those of the intact knee.

Insertion of the PCL-retaining TKR caused a number of significant changes in the kinematics and forces. Adduction increased significantly across the flexion range. The patellar tendon angle was significantly different from that of the intact knee and the UKR, with much less change over the range of tibiofemoral flexion (Fig. 4). The overall pattern of patellar flexion was unaffected (Fig. 1), but there were significant changes in tilt, with the patella tilting more medially than in the intact knee and the UKR (Fig. 2). Patellar spin was more external in the TKR than in the intact knee or the UKR in extension (Fig. 3); spin showed a sudden jump to a more normal internal position at $50^{\circ}$ of tibiofemoral flexion. There were also sudden changes in the point of application of the patellofemoral force on the patella, in the mediolateral component of this force (Fig. 6), and in the quadriceps force (Fig. 5) near $60^{\circ}$ of flexion.

\section{Discussion}

Following insertion of the mobile-bearing UKR, patellar kinematics were found to be very similar to those of the intact knee, with few differences lying outside the error of the method of measurement employed. The only clear difference was an offset of $2^{\circ}$ in the mean value of patellar spin across the range of extension (Fig. 3). The changes in all three patellar rotations across the range of movement (Figs 1 to 3 ) were indistinguishable from those of the intact specimen, suggesting that patellar tracking was occurring normally. The patellar tendon angle remained normal (Fig. 4), supporting the findings from previous in vitro and in vivo studies of this device. ${ }^{13,24}$ Tibiofemoral abductionadduction was also unchanged, and the normal coupling of axial and coronal rotation to knee flexion was maintained, although the mean total tibial rotation was reduced by a few degrees compared with the normal knee.

Implantation of the mobile-bearing UKR did not have a significant effect on the pattern of change in patellofemoral or quadriceps force across the range of knee flexion. The 
peak patellofemoral compressive force was reduced by approximately $5 \%$ and the quadriceps force was increased by a similar amount at between $80^{\circ}$ and $100^{\circ}$ of flexion (Fig. 5). Importantly, the ratio of these two forces was similar to that for the intact knee, implying that the inter-relationship of the orientation of the patellar tendon and quadriceps tendon, which dictates their force ratio, remains normal. The point of action of patellofemoral force was also unchanged over the flexion range, consistent with the proximal migration of this point seen in the normal knee. The components of patellofemoral shear force (Figs 6 and 7) were not significantly altered.

The near-normal patellofemoral kinematics of the mobile-bearing UKR are in contrast to some of the abnormal movements and forces seen after implantation of the PCL-retaining TKR. In the latter case, patellar spin and tilt were significantly disturbed, demonstrating abnormal tracking of the patella. Patellar tilt was abnormal throughout the majority of the range of knee flexion, with $10^{\circ}$ of abnormal medial tilt in full flexion (Fig. 2). As the knee extended, a dramatic change in patellar spin occurred at approximately $50^{\circ}$ of flexion (Fig. 3). This corresponded to the point where the patella, after articulating with the prosthetic condyles, engaged with the trochlear groove of the TKR prosthesis. Although the change in patellar flexion remained normal over most of the range of knee flexion, a difference was seen during the transition from condylar to trochlear articulation (Fig. 1). At this point, a decrease in the angle of patellar flexion occurred, suggesting that as the knee extended the patella did not glide smoothly on to the trochlea. Accompanying these altered kinematics were changes in quadriceps force (Fig. 5), patellofemoral compressive force (Fig. 5) and patellofemoral mediolateral shear force (Fig. 6). A possible explanation for this is the impingement of the anterior aspect of the patella on the trochlear groove, causing the patella to hinge around its superior pole, restricting patellar flexion. This transient change in patellar orientation also corresponds to an abnormal change in the point of action of the patellofemoral force. As the knee extends further, the patella is released and returns to a more normal orientation. This subtle change in orientation was of insufficient magnitude to be noticed at insertion of the implant, when patellar tracking was assessed. It is only evident with more sensitive measurement of patellar kinematics and forces. This type of patellar 'catch' has been reported previously in clinical series of other total knee and patellofemoral replacements. ${ }^{25,26}$ The 'catch' was not seen with insertion of the UKR. Although the posterior femoral condyle is replaced in a medial mobile-bearing UKR, the trochlear groove is retained, and this combination allows the smooth transition of the patella from the condyles on to the trochlea. ${ }^{27}$ Maintaining normal transition from patellocondylar to patellotrochlear articulation is likely to be an important component of good patellofemoral function. Impingement of the patella on the femoral prosthesis and progression of arthritis in the retained patellofemoral joint have been seen with some fixed-bearing unicompartmental prostheses, ${ }^{28,29}$ but have not been described with the Oxford device. ${ }^{14,18,30,31}$

Having discussed the findings for individual variables, it is also important to reflect on the results as a whole, as the tibiofemoral and patellofemoral joints function with significant interdependence. Following insertion of the medial mobile-bearing UKR, all parameters investigated showed little change, providing strong evidence that the knee, and in particular the extensor mechanism, functions normally. This finding agrees with the work of Patil et al, ${ }^{32}$ who also noted that a fixed-bearing UKR preserved normal tibiofemoral kinematics and quadriceps forces. In contrast, insertion of a TKR produced significant abnormalities in many aspects of patellofemoral function, a finding supported by other kinematic studies. ${ }^{33}$ It is important to consider how the mobile-bearing UKR functions. During insertion, much of the normal joint structure is preserved. The lateral compartment and the trochlear groove of the femoral condyles remain unchanged. However, the posterior femoral condyle is replaced, and the patella is in contact with this for approximately $40 \%$ of the range of flexion; hence, a potential for introducing abnormality exists. Despite this, the prosthetic condyle permits a normal change in patellar flexion, tilt and spin, indicating that the replaced part does not significantly alter the geometry of the distal femur. Furthermore, the function of the patellofemoral joint cannot be seen in isolation, as it is affected by movements of the tibiofemoral joint. The use of a flat tibial plateau is not anatomical, but, as predicted by computer modelling, the chosen geometry and use of a meniscal bearing appear to allow normal translation in the sagittal plane, together with a near-normal pattern of tibial rotation. ${ }^{27}$ This is an essential component in retaining normal patellofemoral kinematics.

This study highlights the abnormalities that can occur in knee function following implantation of a TKR. In particular, the extensor mechanism is vulnerable to the knock-on effect of abnormal tibiofemoral movement, with resultant changes in patellar forces and tracking. Insertion of a mobile-bearing unicompartmental device appears to produce very few changes to the extensor mechanism function. This may help to explain the low incidence of patellofemoral complications and excellent patient function reported with its use. ${ }^{14,18,30,31,34}$

The authors would like to thank Mr Adrian Weale for his help in acquiring the patellar transducer used in this study. This research was supported by the Wishbone Trust (UK) and Biomet Inc. Paul Oppold held a Marshall Scholarship funded by the Marshall Aid Commission.

The author or one or more of the authors have received or will receive benefits for personal or professional use from a commercial party related directly or indirectly to the subject of this article. In addition, benefits have been or will be directed to a research fund, foundation, educational institution, or other nonprofit organisation with which one or more of the authors are associated.

\section{References}

1. Walker PS. Requirements for successful total knee replacements: design considerations. Orthop Clin North Am 1989;20:15-29. 
2. Goodfellow J, O'Connor J. The mechanics of the knee and prosthesis design. J Bone Joint Surg [Br] 1978;60-B:358-69.

3. Banks SA, Harman MK, Hodge WA. Mechanism of anterior impingement damage in total knee arthroplasty. J Bone Joint Surg [Am] 2002;84-A(Suppl 2):37-42.

4. Banks SA, Markovich GD, Hodge WA. The mechanics of knee replacements during gait: in vivo fluoroscopic analysis of two designs. Am J Knee Surg 1997;10:261-7.

5. Browne C, Hermida JC, Bergula A, Colwell CW Jr, D’Lima DD. Patellofemoral forces after total knee arthroplasty: effect of extensor moment arm. Knee 2005;12:81 8.

6. Komistek RD, Dennis DA, Mabe JA, Walker SA. An in vivo determination of patellofemoral contact positions. Clin Biomech 2000;15:29-36.

7. Komistek RD, Scott RD, Dennis DA, et al. In vivo comparison of femorotibial contact positions for press-fit posterior stabilized and posterior cruciate-retaining total knee arthroplasties. J Arthroplasty 2002:17:209-16.

8. StiehI JB, Dennis DA, Komistek RD, Crane HS. In vivo determination of condylar lift-off and screw-home in a mobile-bearing total knee arthroplasty. J Arthroplasty 1999; 14:293-9.

9. Stiehl JB, Dennis DA, Komistek RD, Keblish PA. In vivo kinematic analysis of mobile bearing total knee prosthesis. Clin Orthop 1997;345:60-6.

10. Rand JA. Extensor mechanism complications following total knee arthroplasty. $J$ Bone Joint Surg [Am] 2004;86-A:2062-72.

11. Goodfellow J. Knee prostheses: one step forward, two steps back. J Bone Joint Surg [Br] 1992;74-B:1-2.

12. Imran A. Mechanics of the ligament deficient knee. Department of Engineering Science. Oxford University. DPhil 1998.

13. Miller RK, Goodfellow JW, Murray DW, O'Connor JJ. In vitro measurement of patellofemoral force after three types of knee replacement. J Bone Joint Surg $[\mathrm{Br}]$ 1998;80-B:900-6.

14. Emerson RH Jr, Hansborough T, Reitman RD, Rosenfeldt W, Higgins LL. Comparison of a mobile with a fixed-bearing unicompartmental knee implant. Clin Orthop 2002; $404: 62-70$

15. Kumar A, Fiddian NJ. Medial unicompartmental arthroplasty of the knee. The Knee 1999;6:21-3.

16. Lewold S, Goodman S, Knutson K, Robertsson O, Lidgren L. Oxford meniscal bearing knee versus the Marmor knee in unicompartmental arthroplasty for arthrosis: a Swedish multicenter survival study. J Arthroplasty 1995;10:722-31.

17. Murray DW, Goodfellow JW, O'Connor JJ. The Oxford medial unicompartmental arthroplasty: a ten-year survival study. J Bone Joint Surg [Br] 1998;80-B:983-9.

18. Price AJ, Waite JC, Svard U. Long-term clinical results of the medial Oxford unicompartmental knee arthroplasty. Clin Orthop 2005:435:171-80.
19. Robertsson O, Knutson K, Lewold S, Lidgren L. The routine of surgical management reduces failure after unicompartmental knee arthroplasty. J Bone Joint Surg [Br] 2001;83-B:45-9.

20. Singerman R, Berilla J, Kotzar G, Daly J, Davy DT. A six-degree-of-freedom transducer for in vitro measurement of patellofemoral contact forces. $J$ Biomech 1994;27:233-8.

21. Grood ES, Suntay WJ. A joint coordinate system for the clinical description of three-dimensional motions: application to the knee. J Biomech 1983;105:136-44.

22. Zavatsky A, Oppold P, Price A. Simultaneous in vitro measurement of patellofemoral kinematics and forces. J Biomech Eng 2004;126:351-6.

23. Bland M. An introduction to medical statistics. Third ed. Oxford; Oxford University Press. 2000: 175-6.

24. Price AJ, Rees JL, Beard DJ, et al. Sagittal plane kinematics of a mobile-bearing unicompartmental knee arthroplasty at 10 years: a comparative in vivo fluoroscopic analysis. J Arthroplasty 2004;19:590-7.

25. Shoji H, Shimozaki E. Patellar clunk syndrome in total knee arthroplasty without patellar resurfacing. J Arthroplasty 1996;11:198-201.

26. Amis A, Senavongse W, Darcy P. Biomechanics of patellofemoral joint prostheses Clin Orthop 2005;436:20-9.

27. O'Connor JJ, Goodfellow JW, Imran A, et al. Kinematics and mechanics of the knee with application to unicompartmental replacement and the pathomechanics of anteromedial osteoarthritis. In: Cartier P, Epinette JA, Deschamps G, Hernigou P, eds Unicompartmental knee arthroplasty. First ed. Paris; Expansion Scientifique Francaise, 1997:12-27.

28. Hernigou P, Deschamps G. Patellar impingement following unicompartmenta arthroplasty. J Bone Joint Surg [Am] 2002;84-A:1132-7.

29. Berger RA, Meneghini RM, Sheinkop MB, et al. The progression of patellofemoral arthrosis after medial unicompartmental replacement: results at 11 to 15 years. Clin Orthop 2004;28:92-9

30. Rajasekhar C, Das S, Smith A. Unicompartmental knee arthroplasty: 2- to 12-year results in a community hospital. J Bone Joint Surg [Br] 2004;86-B:983-5.

31. Vorlat $\mathbf{P}$, Verdonk $\mathbf{R}$, Schauvlieghe $\mathbf{H}$. The Oxford unicompartmental knee prosthesis: a 5-year follow-up. Knee Surg Sports Traumatol Arthrosc 2000;8:154-8.

32. Patil S, Colwell CW Jr, Ezzet KA, D'Lima DD. Can normal knee kinematics be restored with unicompartmental knee replacement? J Bone Joint Surg [Am] 2005;87A:332-8

33. Stiehl JB, Komistek RD, Dennis DA, Keblish PA. Kinematics of the patellofemoral joint in total knee arthroplasty. J Arthroplasty 2001;16:706-14.

34. Pandit H, Jenkins C, Barker K, Dodd CA, Murray DW. The Oxford medial unicompartmental knee replacement using a minimally-invasive approach. J Bone Joint Surg [Br] 2006;88-B:54-60. 